\title{
Influence of Androgen Receptor Antagonist MDV3100 Therapy on Rats With Benign Prostatic Hyperplasia
}

\author{
Minggen Yang, Zhenqiang Xu, Zhiming Zhuang \\ Department of Urology, Zhangzhou Affiliated Hospital of Fujian Medical University, Zhangzhou, China
}

Purpose: To probe the effect and mechanism of androgen receptor antagonist MDV3100 on benign prostatic hyperplasia $(\mathrm{BPH})$ of rats

Methods: BPH rat model was induced by testosterone propionate. Then antagomir-miR-21-3p or agomir-miR-21-3p was injected into rats before MDV3100 treatment. The prostate index was measured by weighing the wet weight of the rat prostate. The structural morphology of rat prostate was observed after hematoxylin \& eosin staining. Immunohistochemistry was applied to evaluate the expression levels of Ki-6 and inflammatory cytokines (interleukin [IL]-6, IL-18, and tumor necrosis factor- $\alpha$ ) in rat prostate tissues. Quantitative reverse transcription polymerase chain reaction was utilized for assessment of miR-21-3p expression, and Western blot for the performance of the phosphorylation levels of IKKa and p65.

Results: Injection of testosterone propionate caused increased prostate gland hyperplasia, heightened miR-21-3p level, and activated nuclear factor-kappa B (NF-kB) signaling pathway. Additionally, BPH was accompanied by inflammatory response, as evidenced by enhanced expressions of Ki-67 and inflammatory cytokines. MDV3100 exposure ameliorated BPH and suppressed miR-21-3p expression. Overexpression of miR-21-3p intensified BPH and inflammation level, while knockdown of miR-21-3p relieved BPH. The coeffect of miR-21-3p upregulation and MDV3100 subjection led to higher inflammatory response, elevated phosphorylation levels of IKKa and p65 than MDV3100 treatment alone.

Conclusions: Androgen receptor antagonist MDV3100 alleviates BPH and inflammatory response through miR-21-3p downregulation and NF-kB signaling pathway blockade.

Keywords: Androgen receptor antagonist; Benign prostatic hyperplasia; MiR-21-3p; MDV3100; NF-кB pathway; Ki-67

- Fund/Grant Support: This work was supported by Natural Science Foundation of Fujian Province (2017J01381).

- Research Ethics: The experimental scheme was authorized by the Committee of Experimental Animals of Zhangzhou Affiliated Hospital of Fujian Medical University (ZYL No. 2020KYB133). All procedures were in compliance with the Guide for the Care and Use of Laboratory Animals.

- Conflict of Interest: No potential conflict of interest relevant to this article was reported.

\section{- HIGHLIGHTS}

-AR antagonist MDV3100 inhibits BPH in rats.

-MiR-21-3p regulates NF-KB pathway to influence BPH rats.

-MDV3100 suppresses BPH in rats by repressing miR-21-3p.

\section{INTRODUCTION}

Benign prostatic hyperplasia (BPH) is the most frequent diag- nosed benign neoplasm of aged men and is infrequent before age of 40 , but its prevalence increases sharply after age of 40 , reaching $40 \%-50 \%$ at the age of $50-60$ and $80 \%-90 \%$ after the

Corresponding author: Zhenqiang Xu (D) https://orcid.org/0000-0002-3328-9547 Department of Urology, Zhangzhou Affiliated Hospital of Fujian Medical University, No. 59 Victory West Road, Zhangzhou, Fujian 363000, P.R. China Email: xuzhenqiang53@126.com

Submitted: January 8, 2021 / Accepted after revision: April 15, 2021 
age of $80[1,2]$. BPH is a prevailing reason for lower urinary tract symptoms in men, featured by hyperplasia of prostatic stromal and epithelial [3]. Both prostate cancer and BPH are part of the metabolic syndrome, and inflammation elicits a dominating role in the progression of both $\mathrm{BPH}$ and prostate cancer [4]. Traditional cancer therapies including chemotherapy, operation, and radiotherapy, may induce various side effects that destroy the immune systems or kill normal cells of the patients [5]. Thus, there is a pressing need for the selection of the most suitable treatment regimen for the improvement of the patients with $\mathrm{BPH}$. Numerous studies have displayed that the androgen receptor (AR) plays a critical role in the initiation and maintenance of prostate cancer [6,7], and inhibition of AR is an effective option for the treatment of advanced disease [8]. Enzalutamide (formerly MDV3100) is an AR antagonist that is frequently used and offers great promise for patients with prostate cancer $[9,10]$. Nevertheless, the detailed mechanism of AR antagonist MDV3100, especially the pathogenic roles of MDV3100 in BPH, is still largely unearthed.

Nuclear factor-kappa B (NF-kB) transcription factors are conserved regulators of inflammatory and immune responses [11]. NF- $\kappa B$ strictly mediates the production of proinflammatory cytokines, such as tumor necrosis factor- $\alpha$ (TNF- $\alpha$ ), interleukin-1 $\beta$ (IL-1 $\beta$ ), and IL-6 [12]. A significant finding of a recent study proposed that the NF- $\mathrm{kB}$ signaling pathway is also implicated in the development and progression of prostate cancer [13]. However, the underlying mechanism of the NF- $\kappa B$ pathway on BPH has not been fully elucidated. Therefore, revealing the cellular and molecular levels, as well as the mechanisms, responsible for $\mathrm{BPH}$ are essential for the development of novel treatments.

Representing single-stranded noncoding RNAs of $\sim 22$ nucleotides, microRNAs (miRNAs) alter gene expression at the posttranscriptional level through translational inhibition or mRNA cleavage [14]. Since their pivotal effect on cancer has been gradually verified, miRNAs have emerged as excellent candidates for the development of minimally invasive biomarkers for cancer diagnosis and prognosis [15]. For instance, urine miR21-5p has been documented to be a biomarker in predicting the response of tadalafil for BPH [16]. The miR-21-5p level was heightened in response to the release of inflammatory cytokines, and that miR-21-5p negatively affected cell survival and function [17]. Additionally, it is well recognized that miRNAs are involved in the regulation of NF- $\kappa B$ pathway $[18,19]$. These interactions indicate that NF- $\mathrm{KB}$ and miRNAs can be served as drug therapeutic targets and potential tumor diagnostic biomarkers for clinical cancer treatment [20]. However, whether NF- $\mathrm{KB}$ pathway and miR-21-3p are involved in the modulatory effect of AR antagonist on BPH is still waiting for exposure.

Here, we described that miR-21-3p significantly aggravated $\mathrm{BPH}$ in the rat model, and BPH could be ameliorated after exposure to AR antagonist MDV3100. Through further functional investigations, we found that the NF- $\mathrm{KB}$ pathway is activated during BPH. Injection of MDV3100 was associated with suppressed NF- $\kappa B$ activity and miR-21-3p expression, suggesting the potential role of $\mathrm{AR}$ antagonist in controlling $\mathrm{BPH}$ is achieved through regulating miR-21-3p/NF- $\mathrm{KB}$ axis.

\section{MATERIALS AND METHODS}

\section{Animals}

Wistar male healthy rats ( $\mathrm{n}=64$, weighing 180-220 g, 7 weeks) were supplied by Laboratory Animal Resources, Chinese Academy of Sciences (Shanghai, China) and housed in a specific pathogen-free laboratory with a cycle of 12 hours light/12 hours dark, and food and water were available ad libitum. The experimental scheme was authorized by the Committee of Experimental Animals of Zhangzhou Affiliated Hospital of Fujian Medical University (ZYL No. 2020KYB133). All procedures were in compliance with the Guide for the Care and Use of Laboratory Animals.

To eliminate the influence of testosterone, the rats were subjected to surgical castration under aseptic conditions with their epididymis and testis removed. Three days after surgery, rats were injected with $3 \mathrm{mg} / \mathrm{kg}$ of testosterone propionate subcutaneously for 7 weeks. Rats were correspondingly assigned to following 8 groups $(\mathrm{n}=8)$ : Model group (subcutaneous injection with $3 \mathrm{mg} / \mathrm{kg}$ of testosterone propionate for 7 weeks), MDV 3100 group (7 weeks of testosterone propionate treatment followed by 5 days of $10-\mathrm{mg} / \mathrm{kg}$ MDV3100 by oral administration, MDV3100 diluted in 10\% DMSO, 45\% polyethylene glycol 400, $45 \%$ normal saline), ant-NC group (exposure to testosterone propionate for 7 weeks and received tail vein injection of antagomir-NC for 3 consecutive days during the 7th week), antmiR-21-3p group (subjection to testosterone propionate for 7 weeks and received tail vein injection of antagomir-miR-21-3p for 3 consecutive days during the 7th week), ago-NC group (treatment with testosterone propionate for 7 weeks and received tail vein injection of agomir-NC for 3 consecutive days during the 7th week), ago-miR-21-3p group (testosterone pro- 
pionate injection for 7 weeks prior to agomir-miR-21-3p injection for 3 consecutive days during the 7th week), ago-miR-21$3 \mathrm{p}+\mathrm{MDV} 3100$ group (exposure to testosterone propionate for 7 weeks, agomir-miR-21-3p injection for 3 consecutive days during the 7 th week and 5 consecutive days of oral administration of $10-\mathrm{mg} / \mathrm{kg}$ MDV3100 for 5 consecutive days) and Normal group (set as negative control).

\section{Measurement of Prostate Index}

The rat prostate was obtained and then weighed with an electronic balance (E02140, OHAUS, Switzerland), and prostate index (PI) was calculated. PI = the wet weight of prostate/the body weight of rat.

\section{Hematoxylin \& Eosin Staining}

The prostate tissues of rat were fixed in $10 \%$ formalin, followed by paraffin embedding to make 4 - $\mu$ m-thick slices. The slices were stained with conventional hematoxylin-eosin, and the prostate structure was observed under a light microscope (DMi8, Leica, Germany).

\section{Immunohistochemistry}

The rat prostate slices were de-waxed in xylene prior to ethanol gradient dehydration. Sections were received $10 \mathrm{mM}$ citrate buffer $(\mathrm{pH}, 6.0)$ for antigen retrieval and immersed in 3\% hydrogen peroxide to block the endogenous peroxidase before immunohistochemistry (IHC). Sections were incubated with the primary antibodies against Ki-67 $(2 \mu \mathrm{g} / \mathrm{mL}$, ab15580, Abcam, Cambridge, MA, USA), IL-6 (1:400, ab6672, Abcam), IL-18 (1:2,000, ab223293, Abcam), and TNF-a (1:200, ab270264, Abcam) overnight at $4^{\circ} \mathrm{C}$. Sections were washed with phosphatebuffered saline (PBS) thrice before and after incubation with the secondary antibody. After that, 1-3 minutes of diaminobenzidine color development was terminated. The nucleus of cells was stained by hematoxylin for 3 minutes, and sections were dehydrated, permeabilized, and sealed. Images were acquired using a light microscope (Nikon Eclipse E100, Tokyo, Japan).

\section{Quantitative Reverse Transcription Polymerase Chain Reaction}

Total RNA was extracted from cells or tissues utilizing TRIzo ${ }^{\circledR}$ (Invitrogen, Carlsbad, CA, USA) based on the protocol. The RNA samples were reversed into cDNA with a cDNA synthesis kit (Toyobo, Osaka, Japan) after quantitation by a spectropho- tometer (Shimadzu, Kyoto, Japan). The SYBR Green PCR Kit (Toyobo, Osaka, Japan) was employed for the performance of quantitative real-time PCR on CDNA and PRISM 7300 sequence detection system (Applied Biosystems, Foster City, CA, USA) for determination. The reaction conditions were $95^{\circ} \mathrm{C}$ predenaturation for 5 minutes, followed by 40 cycles of $95^{\circ} \mathrm{C}$ denaturation for 10 minutes, $60^{\circ} \mathrm{C}$ annealing for 10 seconds and $72^{\circ} \mathrm{C}$ extension for 20 seconds. Data analysis adopted the $2^{-\Delta \Delta \mathrm{Ct}}$ method. The amplified primer sequences of each gene and its primers are in Table 1.

\section{Western Blot}

The rat prostate tissues were soaked in RIPA buffer (protease inhibitors and phosphatase inhibitors) for cell lysis, followed by 30 minutes of $13,000 \mathrm{rpm}$ centrifugation at $4^{\circ} \mathrm{C}$. The supernatant was collected for determination of protein concentration using the BCA kit. The protein was treated with sodium dodecyl sulfate-polyacrylamide gel electrophoresis prior to PVDF membrane transference. The membranes were incubated with the primary antibodies against rabbit-derived GAPDH (1: 10,000, ab181602, Abcam), IKKa (1:5,000, ab32041, Abcam), p-IKKa (1:500, ab38515, Abcam), p65 (1:1,000, ab16502, Abcam), and p-p65 (1:2,000, ab86299, Abcam) overnight. Following PBS tween washing thrice, the membranes were incubated with the secondary antibody at room temperature for $30 \mathrm{~min}$ utes. Before exposure to developing liquid for color development, the membranes were washed with PBS for 4 times. Then chemiluminescence imaging analysis system (GE Healthcare, Beijing, China) was applied for picture capture.

\section{Statistical Analysis}

Statistical analysis of the data utilized GraphPad Prism 7.0 (GraphPad Software Inc., La Jolla, CA, USA). T-test was adopted for the comparison between the 2 groups. Comparisons among multiple groups were analyzed by 1-way analysis of variance. $\mathrm{P}$-values of significance were $\mathrm{P}<0.05$, unless indicated

Table 1. Primer sequence information

\begin{tabular}{ll}
\hline Name & \multicolumn{1}{c}{ Primer } \\
\hline miR-21-3p & F: 5'-GTAGCTGACCACAAC-3' \\
& R: 5'-GATTCCTGGGCTCTGTCACC-3' \\
U6 & F: 5'-CTCGCTTCGGCAGCACA-3' \\
& R: 5'-AACGCTTCACGAATTTGCGT-3' \\
\hline
\end{tabular}

F, forward primer; $\mathrm{R}$, reversed primer. 
otherwise in figure legend.

\section{RESULTS}

\section{AR Antagonist Mitigates BPH in Rats}

BHP rat model induced by testosterone propionate, were treated with AR antagonist MDV3100. As depicted in Fig. 1A, the PI of the model group was prominently higher than that of the normal group, and the PI of the MDV3100 group was markedly lower than that of the model group $(\mathrm{P}<0.05)$. hematoxylin \& eosin (H\&E) staining (Fig. 1B) manifested that the tissues in the normal group exhibited regularly arranged glands and glan- dular cavity, and columnar epithelium in monolayer with basal nucleus and obvious stroma between the glands. Rat in model group had saw-shaped glandular cavity, in which protuberant glands, high columnar shaped glandular epithelium and thickened glandular epithelial cells with less cytoplasm, enlarged nucleus, increased mesenchyme, and dilated small blood vessels were noticed. Slightly hyperplasia of prostate gland was observed in the MDV3100 group of rats, which was neatly arranged. Most of the glandular epithelium in the MDV3100 group was columnar epithelial monolayer, and glandular epithelial cells had transparent cytoplasm and round-shaped nuclear.
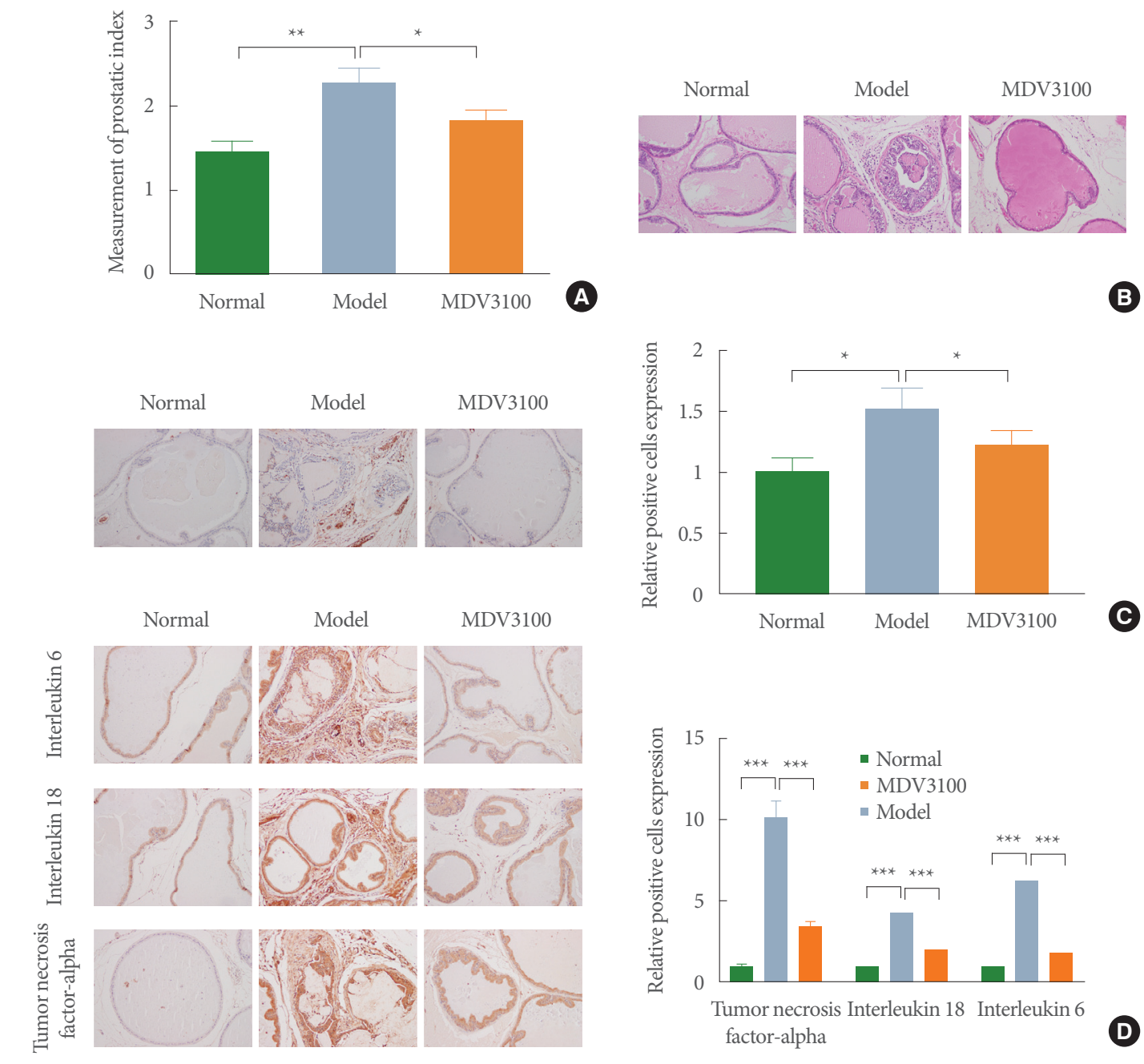

MDV3100
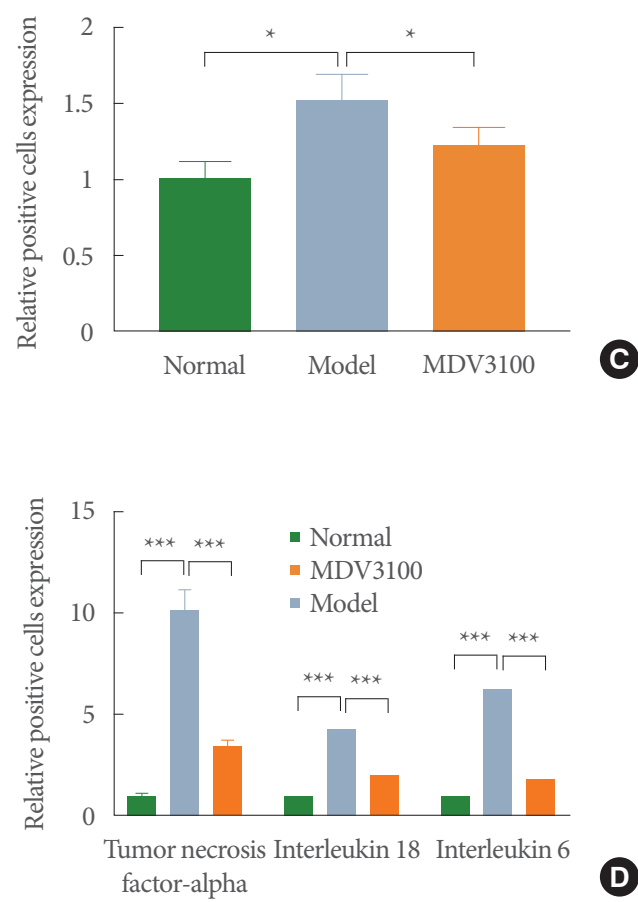

(D)

Fig. 1. Benign prostatic hyperplasia (BPH) in rats can be alleviated by MDV3100. BPH rat models were established by testosterone propionate injection, and rat models were subjected to MDV3100. (A) The prostate index of rats in the different treatment groups was determined. After that, hematoxylin \& eosin staining was utilized for the manifestation of morphology of prostate tissues in rats (B), and immunohistochemistry for assessment of Ki-67 expression (C) as well as inflammatory cytokine levels (D) in prostate tissues; Data were expressed as mean \pm standard deviation. ${ }^{*} \mathrm{P}<0.05 .{ }^{* *} \mathrm{P}<0.01 .{ }^{* *} \mathrm{P}<0.001$. 
Subsequently, the expressions of Ki-67 and inflammatory cytokines in prostate tissues were identified by IHC. Compared with the model group, the normal group and the MDV3100 group possessed lower Ki-67 level (Fig. 1C). Additionally, aggressive increases in expressions of IL-6, IL-18, and TNF- $\alpha$ were found in the model group, and decreases in levels of inflammatory cytokines were discovered in the normal and MDV3100 groups (Fig. 1D). Thus, one explanation for our findings could be that $\mathrm{BPH}$ of rat is accompanied with inflammatory response. AR antagonist MDV3100 exposure interferes with inflammatory response and associates with $\mathrm{BPH}$ amelioration.

\section{NF-KB Signaling Pathway Is Activated in BPH Rats}

We next focused on whether NF- $\kappa \mathrm{B}$ pathway is involved in $\mathrm{BPH}$ in rats. Toward this end, the phosphorylation levels of IKK $\alpha$ and p65 were assessed in rat prostate tissues. Western blot illustrated that the phosphorylation levels of IKKa and p65 in the model group were markedly higher than that of normal group, while these protein expressions in the MDV3100 group were prominently lower than that of model group (Fig. 2A) $(\mathrm{P}<0.05)$. Quantitative reverse transcription polymerase chain reaction (qRT-PCR) on mRNA expression of miR-21-3p exhibited that injection of testosterone propionate led to highly expressed miR-21-3p, and following treatment with MDV3100 restrained miR-21-3p level (Fig. $2 \mathrm{~B})(\mathrm{P}<0.05)$. Taking these data together, $\mathrm{BPH}$ can activate $\mathrm{NF}-\kappa \mathrm{B}$ pathway and heighten expression level of miR-21-3p in rats, while MDV3100 intervention can block the NF- $\kappa \mathrm{B}$ pathway and down-regulate miR21-3p expression in BPH rats.

\section{MiR-21-3p Aggravates BPH in Rats}

We sought to examine whether miR-21-3p is implicated in BPH. Here, antagomir-miR-21-3p or agomir-miR-21-3p was injected into $\mathrm{BPH}$ rats through tail vein. qRT-PCR analysis of miR-21-3p expression displayed that there was increased mRNA level of miR-21-3p in the ago-miR-21-3p group (Fig. $3 \mathrm{~A} ; \mathrm{P}<0.05$, vs. ago-NC group) and decreased miR-21-3p level in the ant-miR-21-3p group (Fig. $3 \mathrm{~A} ; \mathrm{P}<0.05$, vs. ant-NC group). PI measurement yielded that miR-21-3p upregulation raised $\mathrm{PI}$ of rats and miR-21-3p downregulation reduced PI (Fig. 3B) $(\mathrm{P}<0.05)$.

$\mathrm{H} \& \mathrm{E}$ staining observed that treatment with ant-miR-21-3p decreased prostate gland hyperplasia and increased glandular cavity. The glandular epithelium in the ant-miR-21-3p group was mainly in single columnar shape, and glandular epithelial cells possessed round nucleus. However, injection of ago-miR21-3p intensified hyperplasia of prostate glands, and the glandular epithelium protruded into the cavity. Most of the glandular epithelium in the ago-miR-21-3p group was in high columnar shape, and the glandular epithelial cells with round or oval nucleus were obviously hypertrophic (Fig. 3C).

IHC staining presented that in comparison to the ago-NC group, the ago-miR-21-3p group had elevated Ki-67 expression,
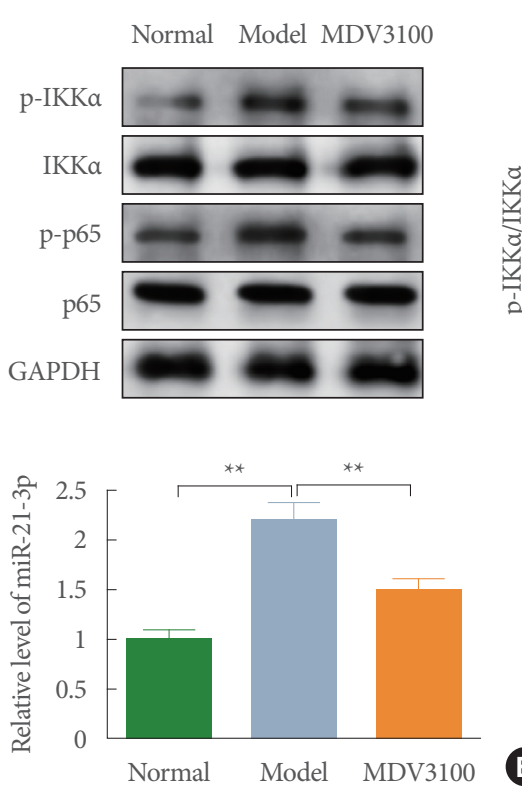

Int Neurourol J September 30, 2021
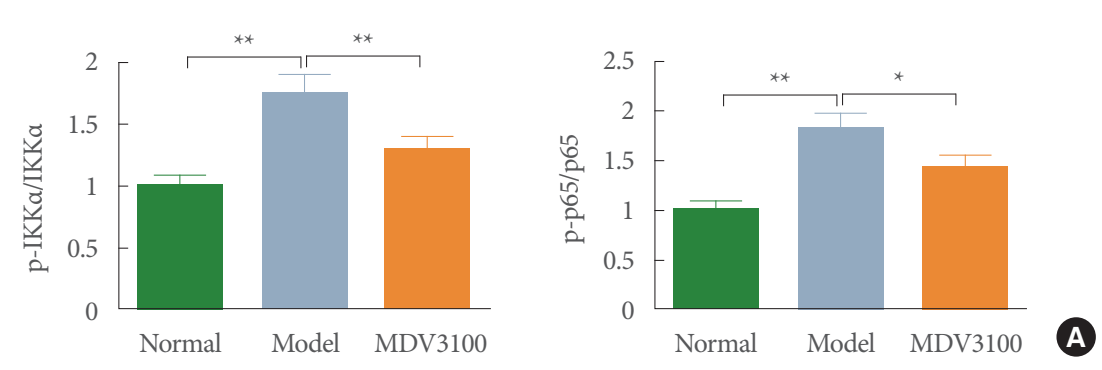

Fig. 2. MDV3100 blocks nuclear factor-kappa B signaling pathway and suppresses miR-21-3p expression in benign prostatic hyperplasia (BPH) rats. Rats were injected with testosterone propionate to construct $\mathrm{BPH}$ model, and then the role of MDV3100 in BPH was explored after rats given to MDV3100. (A) The phosphorylation levels of IKKa and p65 were displayed by Western blot. (B) The mRNA expression of miR-21-3p was evaluated by quantitative reverse transcription polymerase chain reaction. Data were shown as mean \pm standard deviation. ${ }^{\star} \mathrm{P}<0.05$. ${ }^{*} \mathrm{P}<0.01$. 

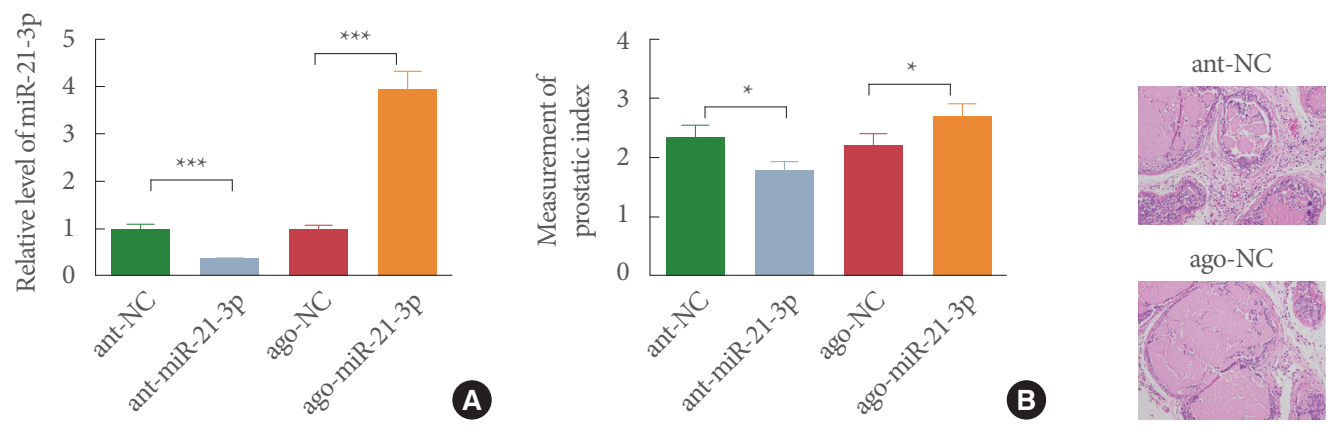

ant-miR-21-3p

(3)
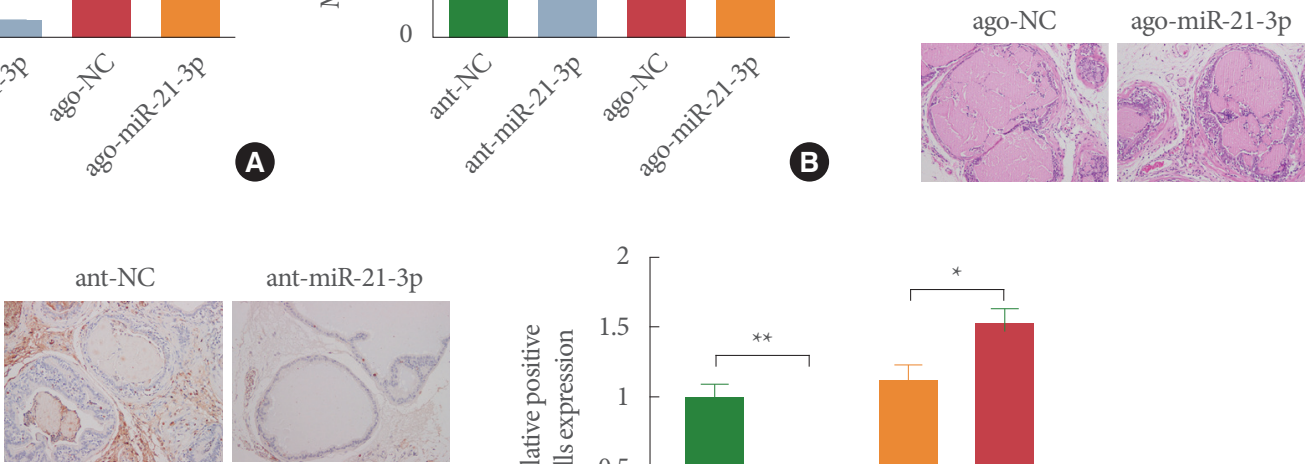

ant-miR-21-3p

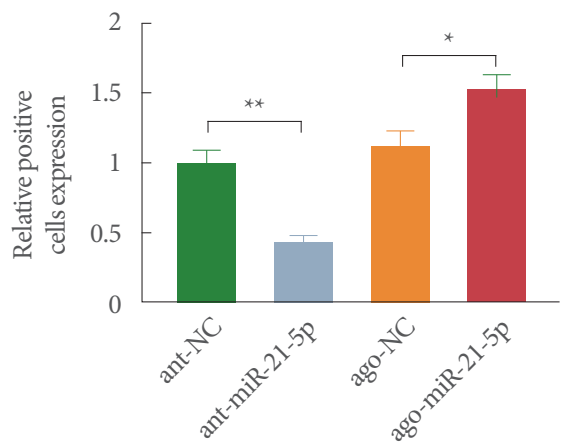

(D)
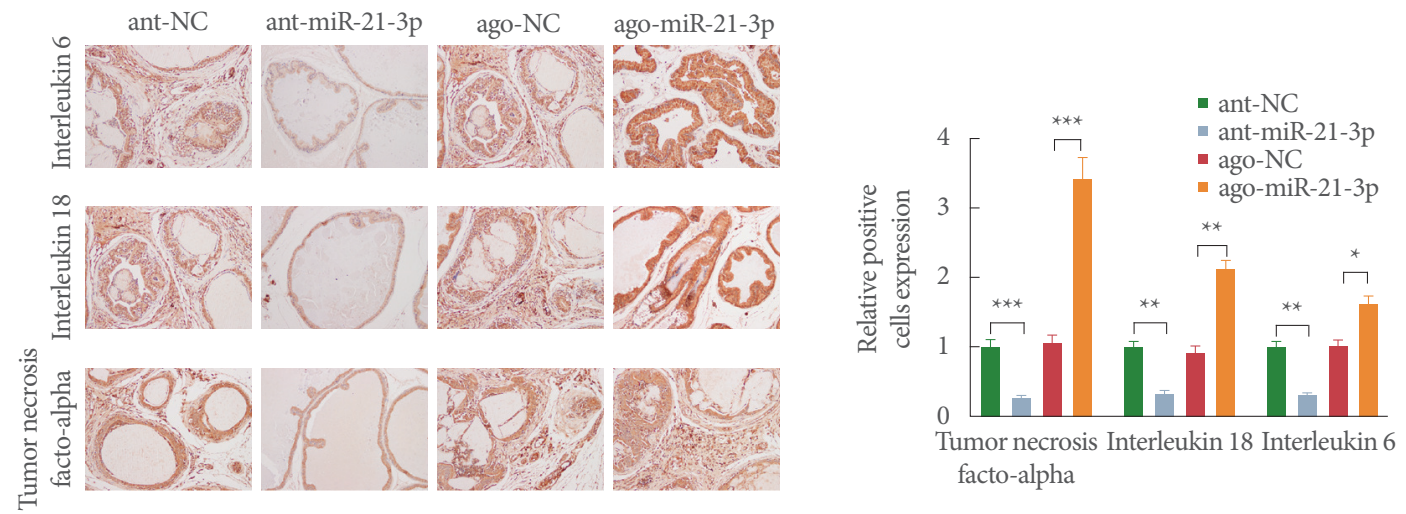

E

Fig. 3. MiR-21-3p interferers with benign prostatic hyperplasia of rats. (A) After tail vein injection of antagomir-miR-21-3p and agomir-miR-21-3p with rats, the mRNA expression level of miR-21-3p was detected by quantitative reverse transcription polymerase chain reaction. The prostate index of rats was determined (B), and structural morphology of prostate tissues was observed after hematoxylin \& eosin staining (C). Immunohistochemistry was used to measure the expression levels of Ki-67 (D), interleukin (IL)-6, IL-18, and tumor necrosis factor- $\alpha(\mathrm{E})$ in rat prostate tissues. Data presented as mean \pm standard deviation. ${ }^{\star} \mathrm{P}<0.05 .{ }^{*} \mathrm{P}<0.01 .{ }^{* *} \mathrm{P}<0.001$.

while compared with the ant-NC group, the ant-miR-21-3p group possessed repressed Ki-67 level (Fig. 3D). Besides, overexpression of miR-21-3p enhanced inflammatory cytokine levels and knockdown of miR-21-3p reduced the expressions of IL-6, IL-18, and TNF- $\alpha$ (Fig. 3E). Collectively, BPH of rats can be intensified by miR-21-3p.

\section{AR Antagonist Improves BPH by Blocking NF-KB Signal Pathway and Suppressing miR-21-3p}

To seek further evidence for the potential synergistic effects of AR antagonist MDV3100 on the regulation of $\mathrm{BPH}, \mathrm{BPH}$ rats were injected with agomir-miR-21-3p. Assessment of qRT-PCR performed that the ago-miR-21-3p+MDV3100 group had a higher expression of miR-21-3p than the MDV3100 group (Fig. $4 \mathrm{~A})(\mathrm{P}<0.05)$. The activation of NF- $\kappa \mathrm{B}$ signaling pathway ana- 

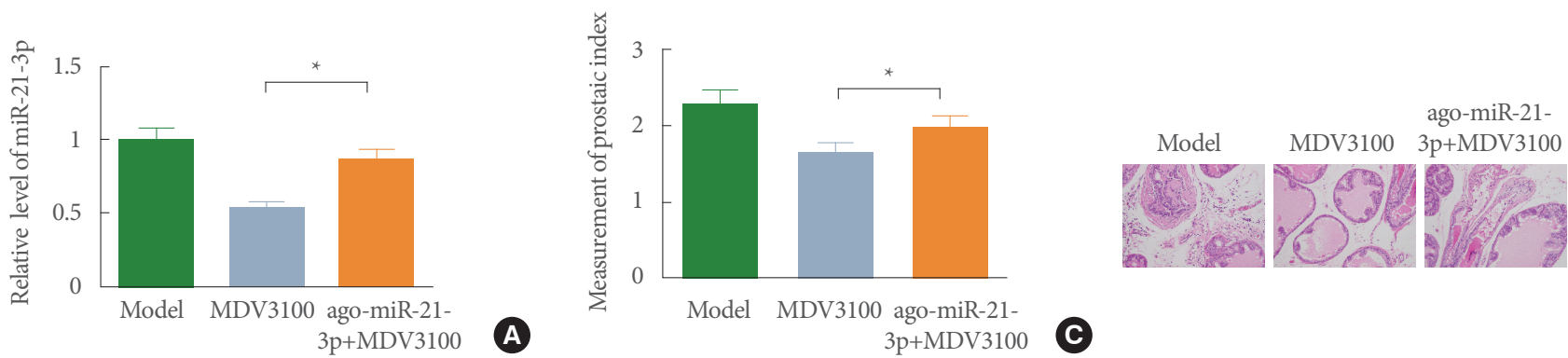

(D)
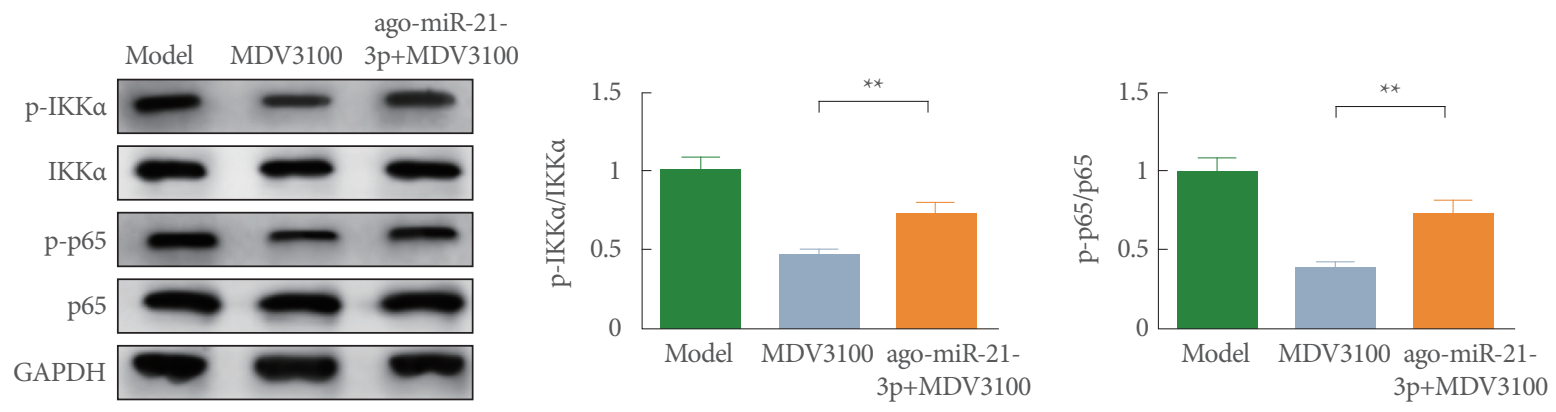

B
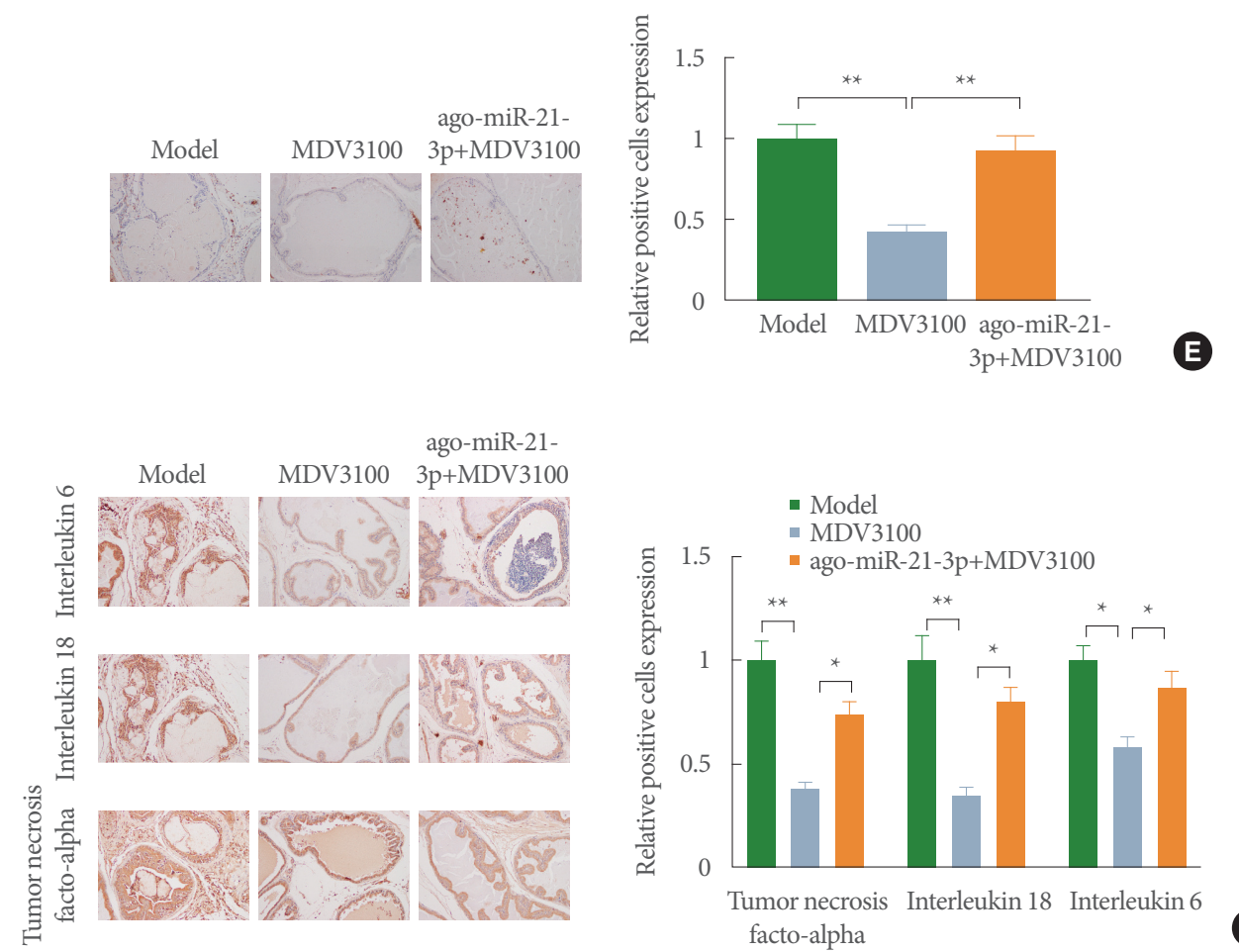

Fig. 4. MDV3100 ameliorates benign prostatic hyperplasia (BPH) of rats by mediation of miR-21-3p and nuclear factor-kappa B signal pathway. BPH rats were given to agomir-miR-21-3p followed by MDV3100 treatment. Quantitative reverse transcription polymerase chain reaction was employed for the detection of miR-21-3p expression (A), and then Western blot for the performance of the phosphorylation levels of IKKa and p65 (B). (C) Prostate index was measured. Hematoxylin \& eosin staining was utilized to observe the morphology of prostate tissues (D), and immunohistochemistry was adopted to assess the expression levels of Ki-67 (E) and inflammatory cytokines $(\mathrm{F})$ in rat prostate tissues. Data expressed as mean \pm standard deviation. ${ }^{\star} \mathrm{P}<0.05 .{ }^{*} \mathrm{P}<0.01$. 
lyzed by Western blot described a prominent rise in the phosphorylation levels of IKKa and p65 in the ago-miR-21-3p+ MDV3100 group rather than the MDV3100 group (Fig. 4B) $(\mathrm{P}<0.05)$.

Additionally, a significantly elevated PI of rats was observed in the ago-miR-21-3p+MDV3100 group over the MDV3100 group (Fig. 4C) $(\mathrm{P}<0.05)$. H\&E staining manifested that compared with the MDV310 group, there was increased prostate gland hyperplasia in the ago-miR-21-3p+MDV3100 group. The glandular epithelium was mainly presented in high columnar shape, and the glandular epithelial cells with round or oval nucleus were hypertrophic (Fig. 4D). Results obtained from IHC staining proposed that the levels of Ki-67 (Fig. 4E), IL-6, IL-18, and TNF- $\alpha$ (Fig. $4 \mathrm{~F}$ ) in rat prostate tissues were enhanced in the ago-miR-21-3p+MDV3100 group than the MDV310 group. Together, these observations suggest that the AR antagonist MDV3100 improves rat BPH through miR-21-3p inhibition and inactivation of NF- $\kappa \mathrm{B}$ signal pathway.

\section{DISCUSSION}

$\mathrm{BPH}$ is a wide-spread chronic condition in elderly males and may significantly affect the quality of life [5,21]. Multiple factors are associated with the pathogenesis of $\mathrm{BPH}$, including chronic inflammation, age-related changes, and disturbance in hormone balance $[22,23]$. A large body of evidence suggests that the majority of patients can achieve satisfactory relief of symptoms with pharmacological therapy [24]. The human AR elicits a major effect on prostate cancer and represents a well-established drug target [8]. Herein, the BPH rat model was constructed by testosterone propionate injection to evaluate whether AR antagonist MDV3100 can mitigate BPH and what is the mechanism under this amelioration.

Enzalutamide is an orally nonsteroidal, available, secondgeneration antiandrogen that acts on many steps in the AR signaling pathway and performs itself as an effective drug for prostate cancer therapy $[25,26]$. In this study, decreased PI, diminished expression of proliferation marker Ki-67, and restrained inflammatory cytokine level were discovered in the $\mathrm{BPH}$ rats following subjection to MDV3100. These findings emphasized that AR antagonist MDV3100 can ameliorate BPH, although the mechanism on AR antagonist interfered with $\mathrm{BPH}$ remains a crucial challenge. The upregulation of miR-21 is reported to be associated with an independent predictor of progress-free survival in patients with advanced prostate cancer
[27]. Besides, AR can be activated by IL-6 to promote cell proliferation in prostate cancer, while miR-21 plays an intermediary role in activating IL- 6 to regulate programmed cell death 4 expression [28]. Here, highly expressed miR-21-3p was found in $\mathrm{BPH}$ rats, and the acquired silence of miR-21-3p emerged following exposure to MDV3100. Thus, we hypothesized that miR-21-3p might participate in AR antagonist-interfering $\mathrm{BPH}$ in the light of recent advances in the understanding of the property of miR-21. To test our hypothesis, the BPH rat model was exposed to antagomir-miR-21-3p or agomir-miR-21-3p by tail vein injection to evaluate the role of miR-21-3p in BPH. Results of H\&E staining, IHC, qRT-PCR, and Western blot verified the effect of miR-21-3p on BPH that overexpression of miR-21-3p intensified BPH and inflammatory response in rats, whereas miR-21-3p knockdown reversed these results.

In this study, elevated phosphorylation levels of NF- $\mathrm{kB}$ pathway-related proteins were found in rats with $\mathrm{BPH}$, which suggests that NF- $\kappa B$ signal pathway was activated in BPH. NF- $\kappa B$ serves as a class of inducible transcription factors that regulate a wide range of genes involved in different processes of the inflammatory and immune responses [29]. Besides, cell survival, invasion, and proliferation in prostate cancer can be facilitated by NF-kB [30]. Here, the levels of NF- $\mathrm{kB}$ pathway-related proteins were discovered to be repressed in response to MDV3100 injection in $\mathrm{BPH}$ rats. With regard to these findings, we next sought to explore whether the NF- $\kappa B$ signaling pathway confers potential synergistic effect with miR-21-3p on AR antagonistinterfering BPH. Support for our hypothesis comes from the observation that the coeffect of miR-21-3p overexpression and MDV3100 treatment could abolish the suppressive effect of MDV3100 on phosphorylation levels of IKKa and p65, and on expressions of IL-6, IL-18, TNF- $\alpha$, and proliferation marker Ki67. Indeed, data is accumulating on the regulatory roles of miR21-3p and the NF- $\kappa B$ pathway in cancers. For example, Ni et al. [31] yielded that CBX7 potentiates stem cell-like characteristics of gastric cancer cells through p16 suppression and the AKTNF- $\kappa B-m i R-21$ pathway activation. Furthermore, miR-21 can effectively restrain the TLR4/NF- $\kappa B$ pathway and myocardial apoptosis in rats with myocardial ischemia-reperfusion [32].

To sum up, our study exhibits the functional role of AR antagonist MDV3100 in testosterone propionate-induced $\mathrm{BPH}$ in rats. MDV3100 can inhibit $\mathrm{BPH}$ and inflammatory response of rats through miR-21-3p repression and the NF- $\kappa B$ signaling pathway blockade. More work is necessary to better understand pathogenesis of $\mathrm{BPH}$ and the deep mechanism which may give 
an insight into the available therapeutic options for the treatment of BPH.

\section{AUTHOR CONTRIBUTION STATEMENT}

- Conceptualization: $M Y, Z X$

- Data curation: $M Y, Z X, Z Z$

- Formal analysis: $M Y, Z Z$

- Funding acquisition: $Z X$

- Methodology: $M Y$

\section{ORCID}

$\begin{array}{ll}\text { Minggen Yang } & 0000-0002-2051-1786 \\ \text { Zhenqiang Xu } & 0000-0002-3328-9547\end{array}$

\section{REFERENCES}

1. Langan RC. Benign prostatic hyperplasia. Prim Care 2019;46:22332.

2. Rastrelli G, Vignozzi L, Corona G, Maggi M. Testosterone and benign prostatic hyperplasia. Sex Med Rev 2019;7:259-71.

3. Lee HN, Lee KS, Lee SY, Shim BS, Lee YS, Hong JH, et al. Effects of doxazosin on alpha 1-adrenergic receptors in prostates with benign prostatic hyperplasia. Low Urin Tract Symptoms 2013;5:82-9.

4. Alcaraz A, Hammerer P, Tubaro A, Schroder FH, Castro R. Is there evidence of a relationship between benign prostatic hyperplasia and prostate cancer? Findings of a literature review. Eur Urol 2009; 55:864-73.

5. Koohi Hosseinabadi O, Behnam MA, Khoradmehr A, Emami F, Sobhani Z, Dehghanian AR, et al. Benign prostatic hyperplasia treatment using plasmonic nanoparticles irradiated by laser in a rat model. Biomed Pharmacother 2020;127:110118.

6. Bluemn EG, Coleman IM, Lucas JM, Coleman RT, Hernandez-Lopez S, Tharakan R, et al. Androgen receptor pathway-independent prostate cancer is sustained through fof signaling. Cancer Cell 2017; 32:474-89.e6.

7. Steinestel J, Luedeke M, Arndt A, Schnoeller TJ, Lennerz JK, Wurm $\mathrm{C}$, et al. Detecting predictive androgen receptor modifications in circulating prostate cancer cells. Oncotarget 2019;10:4213-23.

8. Li H, Hassona MD, Lack NA, Axerio-Cilies P, Leblanc E, Tavassoli P, et al. Characterization of a new class of androgen receptor antagonists with potential therapeutic application in advanced prostate cancer. Mol Cancer Ther 2013;12:2425-35.

9. Benoist GE, van Oort IM, Boerrigter E, Verhaegh GW, van Hooij O,
Groen L, et al. Prognostic value of novel liquid biomarkers in patients with metastatic castration-resistant prostate cancer treated with enzalutamide: a prospective observational study. Clin Chem 2020;66:842-51.

10. Rathkopf D, Scher HI. Androgen receptor antagonists in castration-resistant prostate cancer. Cancer J 2013;19:43-9.

11. Tornatore L, Thotakura AK, Bennett J, Moretti M, Franzoso G. The nuclear factor kappa B signaling pathway: integrating metabolism with inflammation. Trends Cell Biol 2012;22:557-66.

12. Zhu WD, Xu J, Zhang M, Zhu TM, Zhang YH, Sun K. MicroRNA-21 inhibits lipopolysaccharide-induced acute lung injury by targeting nuclear factor-kappaB. Exp Ther Med 2018;16:4616-22.

13. Thomas-Jardin SE, Dahl H, Nawas AF, Bautista M, Delk NA. NFkappaB signaling promotes castration-resistant prostate cancer initiation and progression. Pharmacol Ther 2020;211:107538.

14. Zhang N, Li Z, Bai F, Ji N, Zheng Y, Li Y, et al. MicroRNA expression profiles in benign prostatic hyperplasia. Mol Med Rep 2018; 17:3853-8.

15. Cochetti G, Poli G, Guelfi G, Boni A, Egidi MG, Mearini E. Different levels of serum microRNAs in prostate cancer and benign prostatic hyperplasia: evaluation of potential diagnostic and prognostic role. Onco Targets Ther 2016;9:7545-53.

16. Tanaka T, Minami A, Tashiro K, Yoshida N, Tohda A, Yamakoshi Y, et al. Urine miR-21-5p as a potential biomarker for predicting effectiveness of tadalafil in benign prostatic hyperplasia. Future Sci OA 2018;4:FSO304.

17. Lakhter AJ, Pratt RE, Moore RE, Doucette KK, Maier BF, DiMeglio LA, et al. Beta cell extracellular vesicle miR-21-5p cargo is increased in response to inflammatory cytokines and serves as a biomarker of type 1 diabetes. Diabetologia 2018;61:1124-34.

18. Sirotkin AV, Alexa R, Kisova G, Harrath AH, Alwasel S, Ovcharenko D, et al. MicroRNAs control transcription factor NF-kB (p65) expression in human ovarian cells. Funct Integr Genomics 2015;15: 271-5.

19. Yang Y, Wang JK. The functional analysis of MicroRNAs involved in NF-kappaB signaling. Eur Rev Med Pharmacol Sci 2016;20:176474.

20. Wu J, Ding J, Yang J, Guo X, Zheng Y. MicroRNA roles in the nuclear factor kappa B signaling pathway in cancer. Front Immunol 2018;9:546.

21. Li J, Tian Y, Guo S, Gu H, Yuan Q, Xie X. Testosterone-induced benign prostatic hyperplasia rat and dog as facile models to assess drugs targeting lower urinary tract symptoms. PLoS One 2018;13: e0191469.

22. Kim EH, Larson JA, Andriole GL. Management of benign prostatic 
hyperplasia. Annu Rev Med 2016;67:137-51.

23. Popovics P, Awadallah WN, Kohrt SE, Case TC, Miller NL, Ricke EA, et al. Prostatic osteopontin expression is associated with symptomatic benign prostatic hyperplasia. Prostate 2020;80:731-41.

24. Mobley D, Feibus A, Baum N. Benign prostatic hyperplasia and urinary symptoms: evaluation and treatment. Postgrad Med 2015; 127:301-7.

25. Han J, Zhang J, Zhang W, Zhang D, Li Y, Zhang J, et al. Abiraterone and MDV3100 inhibits the proliferation and promotes the apoptosis of prostate cancer cells through mitophagy. Cancer Cell Int 2019;19:332.

26. Sternberg CN. Enzalutamide, an oral androgen receptor inhibitor for treatment of castration-resistant prostate cancer. Future Oncol 2019;15:1437-57.

27. Guan Y, Wu Y, Liu Y, Ni J, Nong S. Association of microRNA-21 expression with clinicopathological characteristics and the risk of progression in advanced prostate cancer patients receiving androgen deprivation therapy. Prostate 2016;76:986-93.

28. Dong B, Shi Z, Wang J, Wu J, Yang Z, Fang K. IL-6 inhibits the targeted modulation of PDCD4 by miR-21 in prostate cancer. PLoS One 2015;10:e0134366.

29. Liu T, Zhang L, Joo D, Sun SC. NF-kappaB signaling in inflammation. Signal Transduct Target Ther 2017;2:17023.

30. Staal J, Beyaert R. Inflammation and NF-kappaB signaling in prostate cancer: mechanisms and clinical implications. Cells 2018;7:122.

31. Ni SJ, Zhao LQ, Wang XF, Wu ZH, Hua RX, Wan CH, et al. CBX7 regulates stem cell-like properties of gastric cancer cells via p16 and AKT-NF-kappaB-miR-21 pathways. J Hematol Oncol 2018;11:17.

32. Pan YQ, Li J, Li XW, Li YC, Li J, Lin JF. Effect of miR-21/TLR4/NFkappaB pathway on myocardial apoptosis in rats with myocardial ischemia-reperfusion. Eur Rev Med Pharmacol Sci 2018;22:792837. 